\title{
Adipose Tissue and Serum CCDC80 in Obesity and lts Association with Related Metabolic Disease
}

\author{
Óscar Osorio-Conles ${ }^{1,2}$, María Guitart ${ }^{2}$, José María Moreno-Navarrete ${ }^{3}$, Xavier Escoté $e^{1,4}$, Xavier Duran ${ }^{1,4}$, \\ José Manuel Fernandez-Real ${ }^{3}$, Anna María Gómez-Foix ${ }^{1,2}$, Sonia Fernández-Veledo ${ }^{1,4}$ and Joan Vendrell ${ }^{1,4}$ \\ ${ }^{1}$ CIBER de Diabetes y Enfermedades Metabólicas Asociadas (CIBERDEM)-Instituto de Salud Carlos III, Madrid, Spain, \\ ${ }^{2}$ Departament de Bioquímica i Biologia Molecular, Institut de Biomedicina de la Universitat de Barcelona, Facultat de Biologia, \\ Universitat de Barcelona, Barcelona, Spain., 'Service of Diabetes, Endocrinology and Nutrition, Institut d'Investigacio Biomedica \\ de Girona and CIBERobn, Girona, Spain, and ${ }^{4}$ Joan XXIII University Hospital, Rovira i Virgili University IISPV, Tarragona, Spain
}

\begin{abstract}
Coiled-coil domain-containing 80 (CCDC80) is an adipocyte-secreted protein that modulates glucose homeostasis in response to diet-induced obesity in mice. The objective of this study was to analyze the link between human CCDC80 and obesity. CCDC80 protein expression was assessed in paired visceral adipose tissue (VAT) and subcutaneous adipose tissue (SAT) from 10 patients (body mass index range 22.4-38.8 $\mathrm{kg} / \mathrm{m}^{2}$ ). Circulating CCDC80 levels were quantified in serum samples from two independent cross-sectional cohorts comprising 33 lean and 15 obese (cohort 1) and 32 morbidly obese (cohort 2) male patients. Insulin sensitivity, insulin secretion and blood neutrophil count were quantified in serum samples from both cohorts. Additionally, circulating free insulin-like growth factor (IGF)- 1 levels and oral glucose tolerance tests were assessed in cohort 1, whereas C-reactive protein levels and degree of atherosclerosis and hepatic steatosis were studied in cohort 2. In lean patients, total CCDC80 protein content assessed by immunoblotting was lower in VAT than in SAT. In obese patients, CCDC 80 was increased in VAT $(P<0.05)$ but equivalent in SAT compared with lean counterparts. In cohort 1, serum CCDC80 correlated negatively with the acute insulin response to glucose and IGF-1 levels and positively with blood neutrophil count independent of BMI, but not with insulin sensitivity. In cohort 2, serum CCDC80 was positively linked to the inflammatory biomarker $C$-reactive protein $(r=0.46$; $P=0.009$ ), atherosclerosis (carotid intima-media thickness, $r=0.62 ; P<0.001$ ) and hepatic steatosis (analysis of variance $P=0.025$ ). Overall, these results suggest for the first time that CCDC80 may be a component of the obesity-altered secretome in VAT and could act as an adipokine whose circulant levels are linked to glucose tolerance derangements and related to inflammationassociated chronic complications.
\end{abstract}

Online address: http://www.molmed.org

doi: $10.2119 / \mathrm{molmed} .2017 .00067$

\section{INTRODUCTION}

Adipokines are bioactive polypeptides secreted by adipocytes and adipose tissue immune cells (1). Obesity changes the adipokine profile in response to the amount or condition of the adipose organ (2), and altered adipokine secretion is considered a contributing factor for the development of obesity-related disorders such as insulin resistance (3), cardiovascular disease (4) and liver steatosis (5).

The gene coiled-coil domain-containing 80 (CCDC80) was initially identified by differential display as upregulated in brown adipose tissue of bombesin

Address correspondence to Oscar Osorio-Conles, Departament de Bioquímica i Biologia Molecular, -2, Av. Diagonal 645. 08028 Barcelona, Spain. E-mail: oscosocon@gmail.com. Or to Sonia Fernández-Veledo or Joan Vendrell, Joan XXIII University Hospital, Rovira I Virgili University IISPV, Research Unit, First Floor, Room 2 C, Dr. Mallafrè Guasch, 4. 43007 Tarragona, Spain. Phone: 977.29.58.00; Fax: 977.29.58.23; E-mail: sonia.fernandezveledo@gmail.com, jvortega2002@gmail.com. Submitted April 17, 2017; Accepted for Publication August 15, 2017; Published Online (www.molmed.org) August 23, 2017.

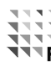
Feinstein Institute
for Medical Research Northwell Health 
that involve Wnt/ $\beta$-catenin signaling, $\mathrm{C} / \mathrm{EBP} \alpha$ and PPAR $\gamma(8)$.

Ccdc80-null mice have normal WAT development but exhibit increased sensitivity to high-fat diet-induced glucose intolerance and reduced glucosestimulated insulin secretion (9). CCDC80 might therefore be a novel modulator of glucose and energy homeostasis. The role of CCDC80 in relation to human obesity is unknown. Rodent (6) and human (10) CCDC80 bear a signal peptide, suggesting that the protein is either expressed on the extracellular surface or secreted. Indeed, secretion of the protein has been demonstrated from primary cultured human adipocytes (7) and differentiated 3T3L1 adipocytes (8). The aim of this study was to investigate the regulation of CCDC80 in human adipose tissue in obesity, and to assess the relationship between circulating CCDC80 and markers of obesity-related metabolic derangements and diseases.

\section{MATERIALS AND METHODS}

\section{Clinical Samples}

All human studies were conducted according to the principles outlined in the Declaration of Helsinki and after approval by local ethical committees. Informed consent was obtained from all individual participants included in the study. Two cohorts from the Hospital Dr. Josep Trueta (Girona, Spain) were used in this study after approval by the Ethics Committee for Clinical Research of the Hospital Universitari de Girona Dr. Josep Trueta. All patients were male. Cohort 1 comprised patients with a wide body mass index (BMI) range $(\mathrm{n}=48, \mathrm{BMI}$ range $20-40 \mathrm{~kg} / \mathrm{m}^{2}$ ). Insulin sensitivity and insulin secretion were studied using the minimal model approach. The following insulin sensitivity indexes were measured and calculated as previously described elsewhere (11): homeostatic model assessment-insulin resistance, fasting insulin resistance index, insulin-glucagon ratio, Raynaud, quantitative insulin sensitivity check index, Bennett's fasting insulin sensitivity index, 2-h insulin/2-h glucose, Gutt insulin sensitivity index 0, 120, composite, Avignon (Sib), Avignon (Si2h) and frequently sampled intravenous glucose tolerance. Insulin secretion was calculated from the frequently samples intravenous glucose tolerance test as the incremental insulin response from 0-10 $\mathrm{min}$ (acute insulin response to glucose, AIRg). Additionally, a $75 \mathrm{~g}$ oral glucose tolerance test (OGTT) was performed following recommendations of the American Diabetes Association. Blood was obtained for determination of glucose and insulin at 0, 30, 60, 90 and $120 \mathrm{~min}$. Serum CCDC80 protein was measured by enzyme-linked immunosorbent assay (ELISA) for human CCDC80 (Cusabio, Wuhan, China), with a $0.078 \mathrm{ng} / \mathrm{mL}$ limit of detection, and intra- and inter-assay coefficients of variation (CVs) of $<8 \%$ and $<10 \%$, respectively. According to the information provided by the supplier, this ELISA uses monoclonal coating and detection antibodies against a fulllength recombinant protein originated from eukaryon expression. Serum-free insulin-like growth factor 1 (IGF1) was measured by immunoradiometric assay (Diagnostic Systems Laboratories, Webster, TX, USA), with an assay sensitivity of $0.80 \mathrm{~g} / \mathrm{L}$ and intra- and inter-assay CVs of $9 \%$ and $11 \%$, respectively. Leukocyte, monocyte and neutrophil counts (EDTA sample; Coulter Electronics, Hialeah, FL, USA) were determined by routine laboratory tests.

Cohort 2 comprised morbidly obese patients $\left(\mathrm{n}=32\right.$, BMI range $35-60 \mathrm{~kg} / \mathrm{m}^{2}$ ) from an ongoing epidemiological study. Inclusion criteria were age 30 to 65 years and $\mathrm{BMI}>35 \mathrm{~kg} / \mathrm{m}^{2}$. Exclusion criteria were systemic diseases, infection in the previous month, serious chronic illness, $>20$ g ethanol intake/day or use of medications that might interfere with insulin action. Furthermore, liver disease (specifically tumoral disease or hepatitis C infection) and thyroid dysfunction were specifically excluded by biochemical workup. Si was measured using the hyperinsulinemic-euglycemic clamp, previously described elsewhere (12).
To measure carotid atherosclerosis, we used a Siemens Acuson S2000 (Mochida Siemens Medical Systems, Tokyo, Japan) ultrasound system with a $3.5 \mathrm{MHz}$ convex transducer to scan the liver and a $7.5 \mathrm{MHz}$ linear array transducer to scan carotid arteries. Images were independently evaluated by two radiologists blinded to clinical and laboratory data according to the Mannheim Consensus (13). Liver biopsies were reviewed by a single pathologist. Hematoxylin and eosin, Masson's trichrome and reticulin stains were performed on each liver sample. Histological features of steatosis, lobular inflammation, hepatocellular ballooning and fibrosis were scored using the non-alcoholic fatty liver disease (NAFLD) scoring system (14). The features were combined to assess the NAFLD activity score (NAS), ranging from 0 to 8 . NAS $\geq 5$ was considered diagnostic of non-alcoholic steatohepatitis, NAS $=0-2$ was considered non-diagnostic of steatohepatitis and NAS $=3-4$ was considered indeterminate. Fibrosis was graded 1-4 and was not included in the activity score.

Roche Hitachi Cobas c711 instrument (Roche, Barcelona, Spain) was used to determine high-density lipoprotein (HDL) cholesterol and total serum triglycerides. HDL cholesterol was quantified by a homogeneous enzymatic colorimetric assay through the cholesterol esterase-cholesterol oxidase-peroxidase reaction (Cobas HDLC3). Serum fasting triglycerides were measured by an enzymatic, colorimetric method with glycerol phosphate oxidase and peroxidase (Cobas TRIGL). LDL cholesterol was calculated using the Friedewald formula.

\section{Adipose Tissue Samples}

Patients were categorized by BMI and classified as lean $\left(\mathrm{BMI}<25 \mathrm{~kg} / \mathrm{m}^{2}\right)$ or obese (BMI $\geq 30 \mathrm{~kg} / \mathrm{m}^{2}$ ) as described (15). Paired visceral adipose tissue (VAT) and subcutaneous adipose tissue (SAT) from 10 patients ( 4 men and 6 women ranging in age from 35 to 70 years), 5 lean (BMI $22.4-24.9 \mathrm{~kg} / \mathrm{m}^{2}$ ) and 5 obese (BMI $30-38.8 \mathrm{~kg} / \mathrm{m}^{2}$ ), were obtained 
from a biobank collection held at the University Hospital Joan XXIII (Tarragona, Spain) after approval by Ethics Committee for Clinical Research of the hospital. All patients were Caucasian, reported steady body weight for at least 3 months prior to the study, were free from any infections in the month preceding the study, were scheduled for an elective surgical procedure (cholecystectomy or surgery for abdominal hernia) and had no metabolic diseases other than obesity. Exclusion criteria were presence of liver or renal disease, malignancy, chronic inflammatory disease or pharmacological treatments that could alter the lipid profile. All patients had fasted overnight for at least $12 \mathrm{~h}$ prior to the surgical procedure.

\section{SGBS Cell Culture}

A human preadipocyte cell line of subcutaneous origin, SGBS (16), was kindly provided by Dr Martin Wabitsch (University of Ulm, Germany). Confluent SGBS preadipocytes were induced to differentiate to mature adipocytes as described (17). Cells were maintained at $37^{\circ} \mathrm{C}$ in a humidified atmosphere of $5 \% \mathrm{CO}_{2}$. Cell monolayers were rinsed in phosphate-buffered saline, frozen in liquid $\mathrm{N}_{2}$ and stored at $-80^{\circ} \mathrm{C}$.

\section{Gene and Protein Expression}

CCDC80 protein levels were analyzed by immunoblotting. Fumarylacetoacetase (FAA) was used as a loading control, since it is expressed in equivalent levels in VAT and SAT and is not regulated by obesity in omental depots (18). Frozen tissue samples were powdered on dry ice and homogenized in a buffer containing 25 mM Tris- $\mathrm{HCl}$ (pH 7.5), $150 \mathrm{mM} \mathrm{NaCl}$, $1 \mathrm{mM}$ EDTA, 1mM EGTA, 1\% Triton X-100, $20 \mathrm{mM}$ sodium pyrophosphate, $25 \mathrm{mM} \mathrm{NaF}, 1 \mathrm{mM} \beta$-glycerophosphate, $1 \mathrm{mM} \mathrm{Na} \mathrm{VO}_{4}$ and $2 \mathrm{mg} / \mathrm{mL}$ leupeptin. SGBS cells were homogenized as described (19). Lysates were gently shaken for $60 \mathrm{~min}$ at $4^{\circ} \mathrm{C}$ and centrifuged at $2500 \mathrm{~g}$ for $10 \mathrm{~min}$ at $4^{\circ} \mathrm{C}$, and supernatants were harvested and stored at $-80^{\circ} \mathrm{C}$ until analysis. Protein was resolved by sodium dodecyl sulfate polyacrylamide gel electrophoresis (8.5\%). Antibodies used were against CCDC80 (R\&D Systems, Minneapolis, MN, USA), CD14 (GenWay Biotech, San Diego CA, USA), FAA (Santa Cruz, Dallas, TX, USA) and GAPDH (Cell Signaling, Beverly, MA, USA). Antibody binding was revealed with horseradish peroxidase-conjugated secondary antibodies (1:1000, Cell Signaling), and membranes were developed with ECL-Plus (GE Healthcare, Buckinghamshire, UK). Protein bands were detected and quantified using an LAS-3000 luminescent image analyzer (Fujifilm, Tokyo, Japan). For CCDC80 quantification, the same whole area covering all detected band sizes was considered.

Total RNA was extracted from SGBS cells as described (19). RNA was retrotranscribed with TaqMan reagents from Applied Biosystems (Carlsbad, CA, USA) using random hexamers and RNase inhibitor. Real-time polymerase chain reaction was performed in a LightCycler 480 with LightCycler 480 Probes Master (Roche Applied Science, Sant Cugat del Valles, Spain). Probes for 18S rRNA and CCDC80 were purchased from Applied Biosystems. The 18S rRNA gene was used as a control to normalize the crossing point. Gene expression levels were estimated by the $2^{-\Delta C P}$ method.

\section{Statistical Analyses}

We used the SPSS/PC+ statistical package (version 16; SPSS, Chicago, IL, USA). For clinical and anthropometrical variables, normally distributed data were expressed as mean values \pm standard deviation or \pm standard error of the mean (SEM), and for variables with a non-Gaussian distribution, values were expressed as the median (25th and 75th percentiles). For statistical analysis of expression variables, values that did not have a Gaussian distribution were logarithmically transformed or analyzed by nonparametric tests. Comparisons between groups were performed by one-way analysis of variance with a post hoc Bonferroni correction, or by a Kruskal-Wallis nonparametric test where appropriate. Associations between quantitative variables were evaluated by Pearson correlation analysis or Spearman correlation for non-normally distributed variables. The independence of the associations was evaluated by linear regression analysis. For AT and cultured cell gene expression experiments, a general linear model repeatedmeasures test was used for the statistical analysis. Statistical significance occurred if a computed two-tailed probability value was $<0.05$.

All supplementary materials are available online at www.molmed.org.

\section{RESULTS}

\section{CCDC80 Protein Levels in Adipose Tissue Depots in Relation to Obesity}

We used immunoblotting to analyze the expression of CCDC80 protein in paired samples of VAT and SAT in a cohort of lean and obese patients. In lean patients, CCDC80 was detected as a major immunoreactive species of approximately $108 \mathrm{kDa}$ (Figure 1A), which is consistent with the molecular weight of the predicted human CCDC80 encoded protein and confirmed by in vitro transcription-translation (10). In obese patients, in addition to the full-length protein, an increased proportion of low-molecular-weight forms (between $50 \mathrm{kDa}$ and $75 \mathrm{kDa}$ ) was also detected, suggesting enhanced proteolysis (Figure 1A). When separately comparing SAT depots, obese patients consistently showed a different pattern, with similar proportions of all protein sizes or mostly smaller forms (50-75 kDa) in all samples analyzed. To a lesser extent, there was also a tendency in VAT depot from obese patients to show higher proportions of lower-molecular-weight forms than lean patients. High-molecular-weight forms (150 kDa) were also negligible (data not shown). CCDC80 protein content (including all species from $50-108 \mathrm{kDa}$ ) was approximately $60 \%$ lower in the VAT depot than in SAT of lean patients (Figure 1B). Total CCDC80 protein 
A

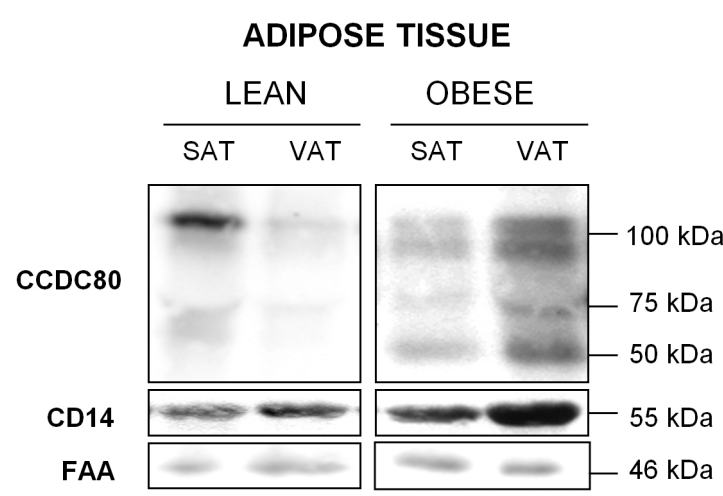

B

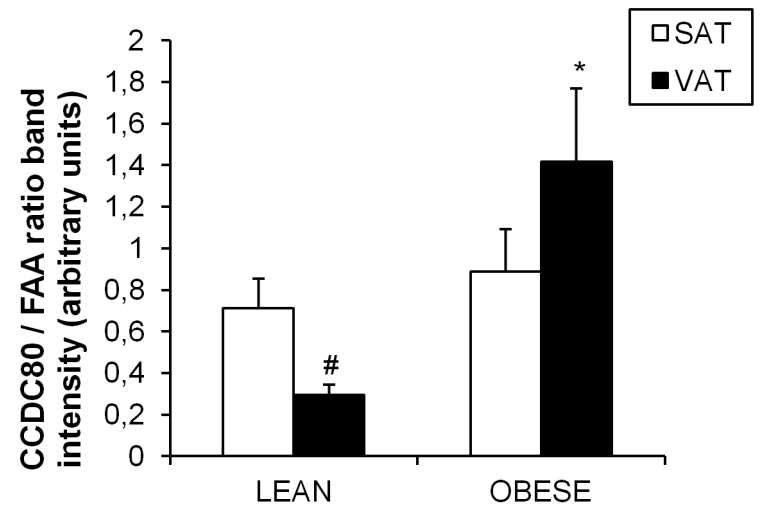

C

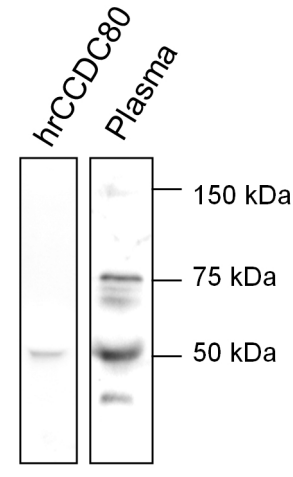

$\mathrm{D}$

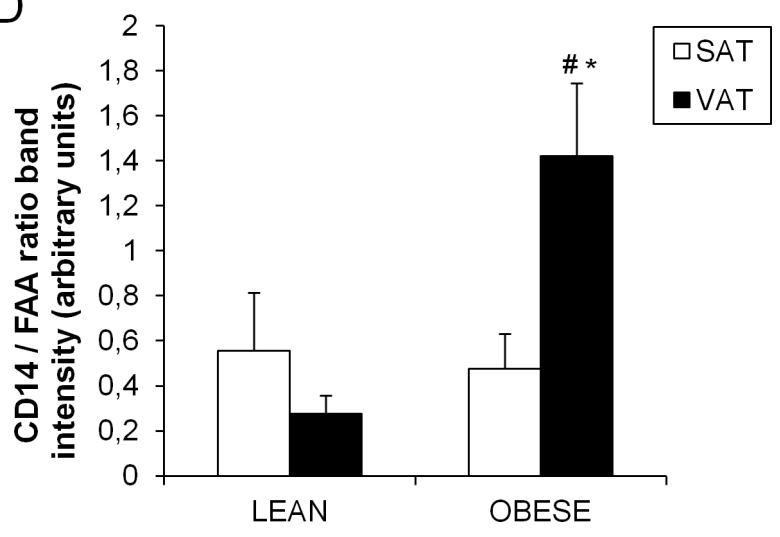

Figure 1. Analysis of CCDC80 and CD14 protein content in SAT and VAT depots from lean and obese patients and CCDC80 protein in human plasma. (A) Western blot analysis of SAT and VAT extracts ( $20 \mu \mathrm{g}$ protein). Membranes were hybridized with antibodies against CCDC80, CD14 and FAA. A representative blot is shown. Bands were quantified and the ratio of intensities between CCDC80 or CD14 and control FAA was calculated. (B) Relative CCDC80 protein content. Data are expressed in arbitrary units and are means \pm SEM from 5 samples. ${ }^{*} P<0.05$ between different depots in lean patients; ${ }^{*} P<0.05$ between VAT depots in obese versus lean patients. (C) Western blot analysis of $1 \mu \mathrm{l}$ of human plasma and $0.8 \mathrm{ng}$ of purified CCDC80 protein (50 kDa fragment). The membrane was hybridized with an antibody against CCDC80. (D) Relative CD14 protein content. Data are expressed in arbitrary units and are means \pm SEM from 5 samples. ${ }^{*} P<0.05$ between different depots in obese patients; ${ }^{*} P<0.05$ between VAT depots in obese versus lean patients.

content (50-108 kDa) was not significantly different between SAT from obese and lean patients; however, CCDC80 protein levels were 3.9-fold higher in VAT depots from obese patients relative to lean counterparts (Figure 1B, $P<0.05$ ). In contrast to adipose tissue expression, analysis of CCDC80 protein in control human plasma by immunoblotting demonstrated a predominance of smaller molecular weight forms (50-75 kDa) and very little expression of the $150 \mathrm{kDa}$ species (Figure 1C).

To evaluate the inflammatory state of adipose tissue, we measured steadystate levels of CD14, a general macrophage marker (20) that is also an inflammation-related gene expressed in adipocytes (21). CD14 protein content relative to FAA was five-fold higher in VAT from obese than lean patients $(P<0.05)$, whereas no significant differences were observed in SAT depots (Figure 1D). Moreover, whereas the relative levels of CD14 were comparable between VAT and SAT depots in lean patients, obese patients exhibited an approximate threefold increase in CD14 expression in VAT relative to SAT $(P<0.05)$.

Thus, obesity was associated with a marked increase in CCDC80 protein content in VAT but not SAT depots, and correlated with an increase in inflammatory CD14 expression.

\section{CCDC80 Expression in Human SGBS Adipocytes}

Although our results showed negligible presence of high-molecular-weight forms of CCDC80 in human adipose tissue depots and a only small amount in control plasma, these species are consistently observed in cultured murine adipocytes (7) and in cells ectopically expressing CCDC80 $(7,22)$. We therefore analyzed total CCDC80 protein in cultured human SGBS cells, in confluent preadipocytes, and in differentiating and mature adipocytes. Adipocyte differentiation was carried out by addition of the adipogenic factors transferrin, insulin, cortisol, triiodothyronine, 
dexamethasone, IBMX and rosiglitazone for $4 \mathrm{~d}$; subsequently, dexamethasone, IBMX and rosiglitazone were withdrawn for the following $10 \mathrm{~d}$. In all stages of differentiation, we detected a major band of approximately $108 \mathrm{kDa}$, corresponding to the full-length protein, as well as lower-molecular-weight species of approximately $75 \mathrm{kDa}$ (Figure 2A). Irrespective of their differentiation stage, abundant forms of higher molecular mass $(150 \mathrm{kDa})$ were also detected in SGBS cells, suggesting extensive protein glycosylation $(10,22)$. The pattern of CCDC80 protein expression detected was consistent with that reported in primary cultured human adipocytes, that is, a robust expression of molecular forms of $75 \mathrm{kDa}$ and $150 \mathrm{kDa}$ (7). Quantification of CCDC80 protein (including all molecular mass forms) relative to GAPDH revealed that differentiating adipocytes had significantly lower levels of CCDC80 compared with confluent preadipocytes after only one day of differentiation, with a minimal expression observed at $6 \mathrm{~d}$ post-differentiation (Figure $2 \mathrm{~A})$. Of note, since GAPDH expression has been found to be substantially decreased in mature, fully differentiated

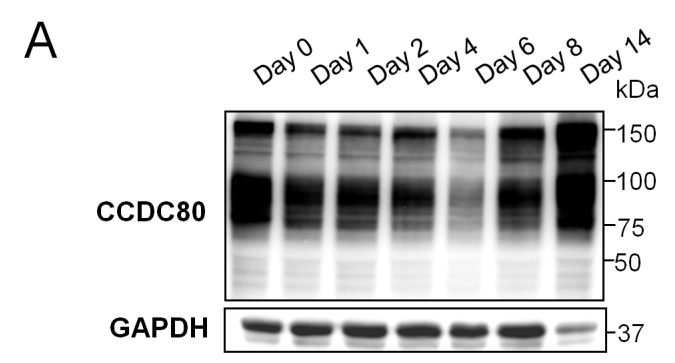

B

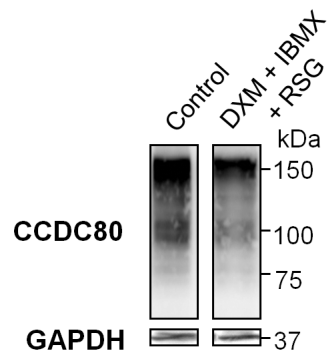

C
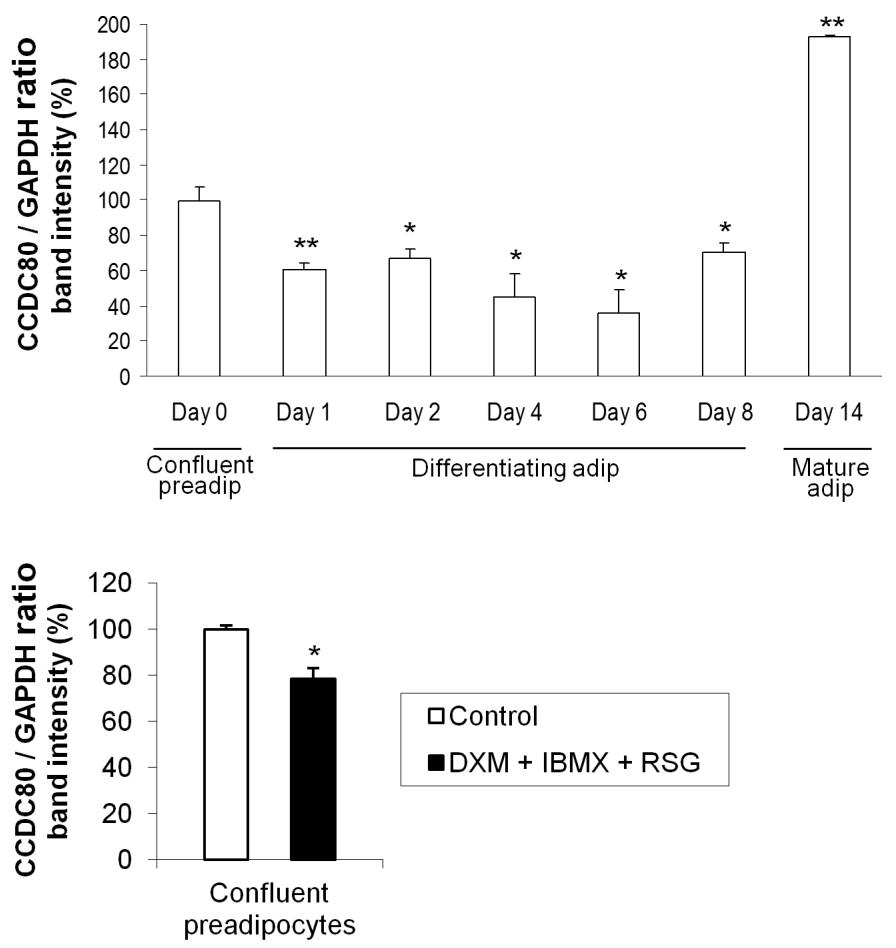

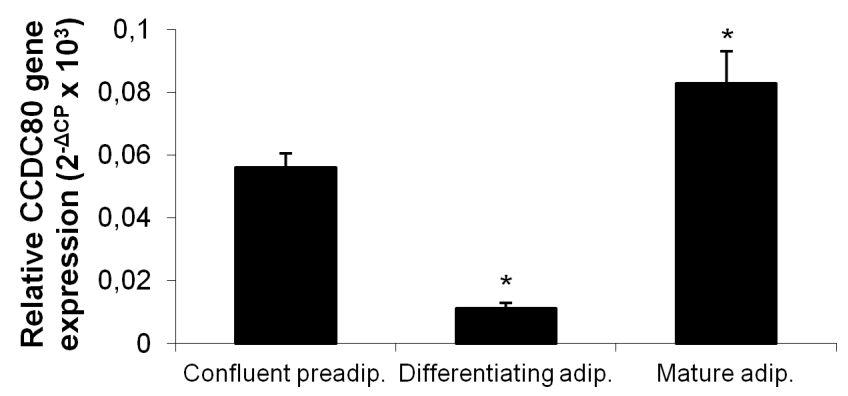

Figure 2. CCDC80 protein expression in human SGBS adipocytes during differentiation. (A) Western blot analysis of CCDC80 protein content in SGBS cell extracts of confluent preadipocytes (d 0), differentiating adipocytes (d 1-8) and mature adipocytes (d 14).

(B) Confluent preadipocytes incubated with or without $25 \mathrm{nM}$ dexamethasone, $0.5 \mathrm{mM}$ IBMX and $2 \mu \mathrm{M}$ rosiglitazone for $16 \mathrm{~h}$. In A and B, a representative image is shown. Ratios of intensity of CCDC80 bands compared with intensity of GAPDH are expressed as a percentage of (untreated) confluent preadipocyte values and are means \pm standard error of the mean from two experiments performed in triplicate. ${ }^{*} P<0.05$ and ${ }^{* *} P<0.01$ vs untreated preadipocytes. (C) CCDC80 mRNA levels relative to 185 rRNA were measured in confluent preadipocytes, differentiating adipocytes (4 d post-differentiation) and mature adipocytes (14 d post-differentiation). Data are means \pm standard error of the mean of $2^{-\Delta \mathrm{CP}} \times 10^{3}$ from two experiments performed in triplicate. ${ }^{*} P<0.001$ to preadipocytes; ${ }^{*} P<0.01$ to differentiating adipocytes. 
SGBS adipocytes (23), the expression of CCDC80 could have been overestimated at differentiation d 14. A similar pattern was observed for CCDC80 mRNA during early-stage differentiation of 3T3L1 cells $(7,8)$, a transient repression that has been associated with the inhibitory effect of the added adipogenic factors dexamethasone and IBMX (8). Indeed, treatment of confluent SGBS preadipocytes for $16 \mathrm{~h}$ with dexamethasone, IBMX and rosiglitazone significantly reduced CCDC80 protein content (Figure 2B). During the later stages of adipocyte maturation (in the absence of dexamethasone, IBMX and rosiglitazone), CCDC80 protein content increased and the greatest amount was detected in mature adipocytes (Figure 2A). Results from mRNA analysis of CCDC80 corroborated the protein expression data and showed that CCDC80 mRNA levels were reduced after differentiation induction and later recovered in mature adipocytes
(Figure 2C). Therefore, CCDC80 expression is transiently downregulated during early stages of adipocyte differentiation and reaches a maximum in mature adipocytes. Moreover, some adipogenic factors may contribute to lower CCDC80 expression during early differentiation.

\section{Relationship of Circulating CCDC80 Protein to Obesity in Humans}

Serum CCDC80 concentrations were quantified by ELISA in a cohort of patients with different degrees of obesity, and there were no differences between lean and obese patients. Clinical and laboratory data for cohort 1 are presented in Table 1. When CCDC80 levels were related to clinical data on insulin secretion and sensitivity, we found that CCDC80 correlated negatively with insulin secretion during the OGTT $(r=-0.35, P=0.02$; Figure $3 \mathrm{~A})$ and with glucose levels $30 \mathrm{~min}$ post-OGTT $(r=-0.34, P=0.02$; Figure 3B). CCDC80

Table 1. Anthropometric and analytical characteristics of the wide body mass index cohort.

\begin{tabular}{|c|c|c|c|}
\hline Cohort 1 & Non-obese & Obese & $P$ \\
\hline $\mathrm{N}$ & 33 & 15 & \\
\hline Age (years) & $52.1 \pm 10.6$ & $52.7 \pm 13.1$ & 0.8 \\
\hline $\mathrm{BMI}\left(\mathrm{kg} / \mathrm{m}^{2}\right)$ & $26.6 \pm 2.4$ & $32.7 \pm 2.1$ & $<0.0001$ \\
\hline Waist circumference $(\mathrm{cm})$ & $89.5 \pm 6.5$ & $106.1 \pm 7.1$ & $<0.0001$ \\
\hline Systolic blood pressure (mmHg) & $124.4 \pm 13.1$ & $140.9 \pm 10.6$ & $<0.0001$ \\
\hline Diastolic blood pressure $(\mathrm{mmHg})$ & $81.3 \pm 8.5$ & $89.2 \pm 12.5$ & 0.01 \\
\hline Total cholesterol (mg/dL) & $212.3 \pm 40.1$ & $215.1 \pm 36.4$ & 0.8 \\
\hline HDL cholesterol (mg/dL) & $56.5 \pm 12.6$ & $53.1 \pm 9.5$ & 0.3 \\
\hline LDL cholesterol (mg/dL) & $135.8 \pm 33.3$ & $2 \pm 34.9$ & 0.9 \\
\hline Fasting triglycerides $(\mathrm{mg} / \mathrm{dL})$ & $78(54.5-114)$ & $91.5(79-157)$ & 0.04 \\
\hline Glucose 0 min post-OGTT (mg/dL) & $96.2 \pm 10.5$ & $102.9 \pm 11.4$ & 0.06 \\
\hline Glucose 30 min post-OGTT (mg/dL) & $171.5 \pm 32.5$ & $184.4 \pm 30.3$ & 0.2 \\
\hline $\mathrm{HbAlc}(\%)$ & $4.8 \pm 0.3$ & $5.0 \pm 0.4$ & 0.2 \\
\hline Fasting insulin $(\mu \mathrm{U} / \mathrm{mL})$ & $8.7 \pm 3.8$ & $14.3 \pm 5.9$ & $<0.0001$ \\
\hline Insulin secretion * & $2.49 \pm 0.42$ & $2.54 \pm 0.40$ & 0.9 \\
\hline Insulin sensitivity ${ }^{\dagger}$ & $0.55 \pm 0.2$ & $0.25 \pm 0.1$ & $<0.0001$ \\
\hline Free IGF-1(ng/mL) & $1.21(0.7-2.1)$ & $0.56(0.3-0.9)$ & 0.1 \\
\hline Neutrophil count $(\mathrm{U} / \mu \mathrm{L})$ & $3522.4 \pm 1175.3$ & $3923.1 \pm 1040.6$ & 0.2 \\
\hline MCP-1 (pg/mL) & $426.7 \pm 139.6$ & $312.6 \pm 105.1$ & 0.02 \\
\hline CCDC80 (ng/mL) & $1.01 \pm 0.48$ & $0.92 \pm 0.45$ & 0.5 \\
\hline
\end{tabular}

*Insulin secretion was measured using acute insulin response to glucose.

'Insulin sensitivity was measured using the frequently sampled intravenous glucose tolerance test.

BMI, body mass index; OGT, oral glucose tolerance test; HbAlc, glycated hemoglobin; IGF-1, insulin-like growth factor-1; MCP-1, monocyte chemoattractant protein-1; CCDC80, coiled-coil domain-containing 80.

was not significantly associated with BMI, insulin sensitivity or age $(P>0.2)$. Interestingly, a negative correlation was found between CCDC80 and circulating levels of free IGF1 $(r=-0.43, P=0.02$; Figure $3 C$ ). We also examined the relationship of circulating CCDC80 with parameters of inflammation and found a positive association with blood neutrophil count $(r=0.26, P=0.07$; Figure $3 D)$. CCDC80 was not significantly associated with dyslipidemia parameters (Supplementary Figure S1A, B).

Circulating CCDC80 protein levels were also assessed in cohort 2 in relation to obesity-associated diseases and inflammation. Anthropometric and analytical characteristics are presented in Table 2. An association was observed between CCDC80 levels and the degree of hepatic steatosis (analysis of variance $P=0.025$; Figure 4A). A correlation was also found between CCDC80 and C-reactive protein $(r=0.46, P=0.009)$. Moreover, a positive correlation was found between CCDC80 and carotid intima-media thickness $(r=0.62, P<0.001$; Figure 4B). Again, CCDC80 was not significantly associated with BMI or age $(P>0.2)$. A positive correlation was found between CCDC80 and fasting triglyceride levels $(r=0.46$, $P=0.01$ ), while no association was found with HDL cholesterol (Supplementary Figure S1C, D).

\section{DISCUSSION}

Adipokines play an important role in adipocyte and systemic metabolic homeostasis. We describe for the first time a differential expression pattern of human CCDC80 molecular-weight forms among adipose tissue depots, and marked changes in CCDC80 expression in obese patients. Moreover, we show that circulating CCDC80 protein levels are associated with glucose disposal and insulin secretion, inflammatory markers, fatty liver disease (FLD) and atherosclerosis.

The human CCDC80 gene is predominantly expressed in WAT $(7,8)$, but its protein pattern of expression in adipose depots and regulation by obesity have 
A

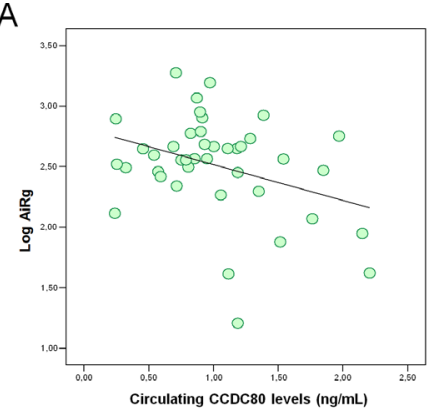

C

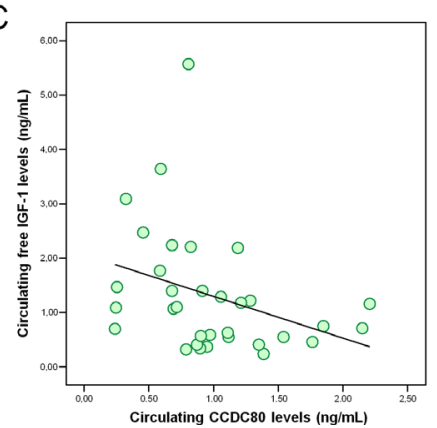

B

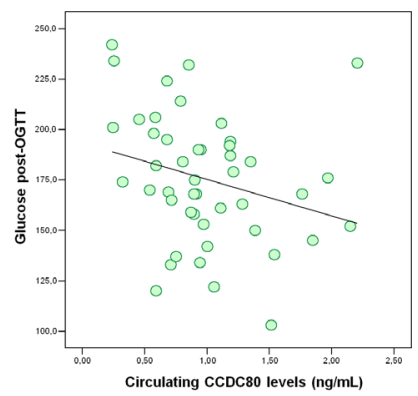

D

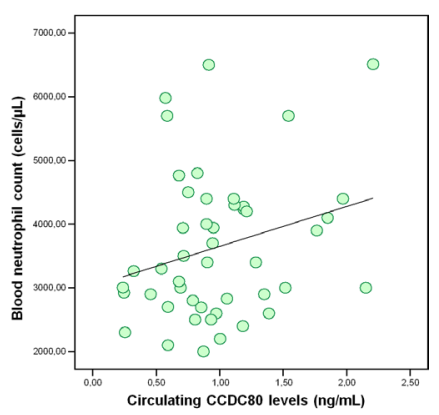

Figure 3. Serum CCDC80 protein levels in a cohort with different degrees of obesity in association with insulin secretion and inflammation parameters. Bivariate correlation analysis showing association of CCDC80 levels with (A) acute insulin response to glucose (AIRg values, which were logarithmically transformed to normalize values), $r=-0.35$, $P=0.02$; (B) with serum glucose levels 30 min post-OGTT, $r=-0.34, P=0.02$; $(C)$ circulating IGF1 levels, $r=-0.43, P=0.01$; and (D) blood neutrophil count, $r=0.26, P=0.07$.

Table 2. Anthropometric and analytical characteristics of the morbidly obese cohort.

\begin{tabular}{ll}
\hline Cohort 2 & All participants \\
\hline $\mathrm{N}$ & 32 \\
Age (years) & $47.8 \pm 8.5$ \\
BMl $\left(\mathrm{kg} / \mathrm{m}^{2}\right)$ & $44.3 \pm 6.3$ \\
Waist circumference $(\mathrm{cm})$ & $124.8 \pm 11.2$ \\
Systolic blood pressure $(\mathrm{mmHg})$ & $139.3 \pm 17.9$ \\
Diastolic blood pressure $(\mathrm{mmHg})$ & $80.3 \pm 13.4$ \\
Total cholesterol $(\mathrm{mg} / \mathrm{dL})$ & $179.1 \pm 29.9$ \\
HDL cholesterol $(\mathrm{mg} / \mathrm{dL})$ & $47.5 \pm 9.8$ \\
LDL cholesterol $(\mathrm{mg} / \mathrm{dL})$ & $108.7 \pm 27.9$ \\
Fasting triglycerides $(\mathrm{mg} / \mathrm{dL})$ & $100(74-144)$ \\
Fasting glucose $(\mathrm{mg} / \mathrm{dL})$ & $113.8 \pm 43.7$ \\
HbAlc $(\%)$ & $6.1 \pm 1.4$ \\
Fasting insulin $(\mu \mathrm{U} / \mathrm{mL})$ & $15.8(8.2-21.9)$ \\
Insulin sensitivity $(\mathrm{mg} /(\mathrm{kg} \cdot \mathrm{min}))^{*}$ & $4.02 \pm 2.5$ \\
Neutrophil count $(\mathrm{U} / \mu \mathrm{L})$ & $4500(3000-6325)$ \\
C-reactive protein $(\mathrm{mg} / \mathrm{dL})$ & $0.7(0.4-1.22)$ \\
CCDC80 (ng/mL) & $1.38(0.95-2.15)$ \\
\hline
\end{tabular}

*Insulin sensitivity was measured using the hyperinsulinemic euglycemic clamp.

$\mathrm{BMI}$, body mass index; HbAlc, glycated hemoglobin; CCDC80, coiled-coil domain-containing 80 .

not previously been determined. SAT and VAT are known to vary in their structural, functional and molecular properties (24), and obesity can also differentially regulate the abundance of particular proteins in either depot (25). In this study, immunoblotting analysis of adipose depots from lean patients demonstrated lower levels of CCDC80 protein in VAT compared with $\mathrm{SAT}$, and also the predominance of the full-length protein. A small proportion of presumably lower-molecular-mass cleavage products (chiefly $75 \mathrm{kDa}$ ) was also observed, whereas expression of the $\mathrm{N}$-glycosylated molecular form (150 kDa) was negligible. This larger molecular species, however, could be detected in cultured adipocytes. In contrast, the total CCDC80 protein content in obese patients was significantly increased in VAT depots compared with lean patients, but was unaltered in SAT depots.

An increased proportion of smaller molecular forms of CCDC80 was also detected in SAT and VAT in obese patients. Secreted CCDC80 associates with the extracellular matrix and is also found in the medium. A substantial portion of it is cleaved. Development of obesity is associated with extensive modifications in adipose tissue involving adipogenesis, angiogenesis and proteolysis of the extracellular matrix $(26,27)$. Proteinases are able to cleave a wide variety of substrates, whereby adipose tissue remodeling may be facilitated, allowing the hypertrophic development of adipocytes observed in obesity. We hypothesize that the different pattern of bands observed in obese relative to lean patients could be due to increased activity of an extracellular proteolytic event rather than experimental bias in obtaining or preparing the samples, which is in agreement with the notion of enhanced extracellular matrix remodeling in obesity. Therefore, CCDC80 displays distinct AT expression patterns and is upregulated by obesity in humans. The result contrasts somewhat with data on rodent models of obesity, where Ccdc 80 mRNA levels are downregulated in 
A

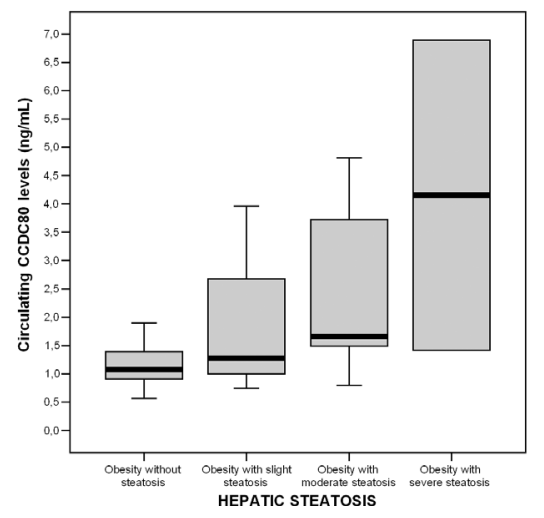

B

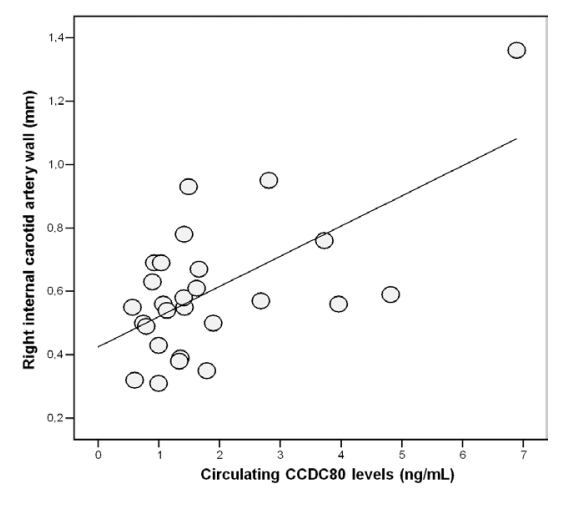

Figure 4. Serum CCDC80 protein levels in a cohort of morbidly obese patients in association with liver steatosis and carotid atherosclerosis. (A) Box plots showing CCDC80 levels (median value and 25th and 75th percentiles) according to degree of hepatic steatosis in consecutive morbidly obese patients without steatosis $(n=11)$ compared with slight $(n=10)$, moderate $(n=5)$ and severe $(n=2)$ steatosis. (B) Bivariate correlation analysis between serum CCDC80 protein levels and internal carotid intima-media thickness.

WAT in $o b / o b$, KKAy and diet-induced obese mice (7), but upregulated in WAT in the mild late-onset obesity model, bombesin receptor subtype-3-deficient mice (6).

It is well recognized that macrophage infiltration and tissue inflammation increase in obesity, particularly in VAT depots (28). CD14 is considered a general macrophage marker (20), although it is also expressed in human adipocytes, where it is upregulated by obesity and tumor necrosis factor-a (21). We found elevated CD14 expression in the VAT depot of obese but not lean patients, whereas no differences were found in SAT. Thus CCDC80 protein levels are increased in VAT in human obesity in parallel with the increased inflammatory state.

Although the precise role of CCDC80 is not yet clear, results from mouse models suggest that CCDC 80 might function to modulate glucose and energy homeostasis $(9,29)$. The high expression of CCDC80 in WAT might point to a role as a local adipose factor inhibiting adipocyte differentiation (29); alternatively, CCDC80 might act as a circulating factor. Previous studies in mice have failed to detect $C C D C 80$ protein in serum (9). Here, CCDC80 immunoblotting of control human plasma revealed major species of $75 \mathrm{kDa}$ and $50 \mathrm{kDa}$ and a minor $150 \mathrm{kDa}$ species. These results are concordant with previous findings of extensive cleavage of the CCDC80 protein extracellularly (8) and secretion of the $150 \mathrm{kDa}$ form (10). Thus, our study demonstrates for the first time that some molecular forms of the CCDC80 protein circulate in human plasma, which suggests that it might elicit systemic actions.

Because the expression of CCDC80 is predominant in $\mathrm{AT}$ and $\mathrm{CCDC} 80$ protein was upregulated in obesity in VAT in parallel with inflammation, we reasoned that circulating CCDC 80 protein could be associated with obesity-linked processes. The concentration of CCDC80 protein in human serum was $0.24-9.1 \mathrm{ng} / \mathrm{mL}$ in the two cohorts combined, comprising a wide BMI range $\left(20-60 \mathrm{~kg} / \mathrm{m}^{2}\right)$. In cohort 1 , with different degrees of obesity (lean to morbidly obese patients), we observed no association between CCDC80 protein and BMI, age or insulin sensitivity. However, serum CCDC80 levels were negatively correlated with AIRg and IGF1 levels. Both low AIRg (30) and IGF-1 levels (31) are related to glucose intolerance. Although a negative correlation of glucose levels at 30 min postOGTT was found, this association was not maintained throughout the test or seen by other insulin sensitivity indexes (Supplementary Table S1). Altogether, this seems to indicate that increased serum CCDC80 is predictive of glucose tolerance derangements.

Interestingly, Ccdc80-null mice on a high-fat diet show exacerbated glucose intolerance and hyperglycemia $(9,29)$, leading to the proposal that CCDC80 might contribute to limiting some of the metabolic dysfunctions associated with diet-induced obesity. However, as the authors point out (29), some criticism can arise from this interpretation, considering that changes in glucose metabolism in ccdc80-null mice could develop secondary to the early increment in fat mass compared with controls, and likely due to loss of inhibition of adipogenesis. Glucose-stimulated insulin secretion in high-fat diets is impaired in Ccdc80-null mice, although the ability of isolated islets to secrete insulin is unaltered (9). No correlations have been found regarding CCDC80 and insulin sensitivity in this study, or in mice (9).

A finding in cohort 1 , with a wide BMI range, was the positive association of CCDC 80 and blood neutrophil count. Obesity is considered a low-grade inflammatory condition linked to some inflammatory mediators, such as neutrophils (32). The fact that circulating CCDC80 was not linked to BMI, but rather to the inflammatory marker, suggests that it reflects the inflammatory condition independent of obesity. Consistent with this, data for cohort 2 (morbidly obese patients) revealed an association with inflammatory and metabolic liver and vascular disease. We found that serum CCDC80 protein levels were associated not only with the inflammatory marker C-reactive protein, but also with the degree of hepatic steatosis. Moreover, carotid intima-media thickness, a marker of atherosclerosis, was also linked to circulating CCDC80 levels. Indeed, FLD and the risk of cardiovascular disease are known to be closely associated with circulating inflammatory markers; for example, C-reactive protein has been associated with liver steatosis independent 
of visceral adiposity in some studies $(33,34)$ and is known to be closely linked to carotid intima-media thickness (35). In addition, nonalcoholic steatohepatitis can predict a more atherogenic risk profile (34). Gene expression of CCDC80 in human liver is insignificant $(7,8)$, and there are no data on the expression of CCDC80 in atherogenic lesions. We speculate that circulating CCDC80 protein either might have a role in these pathogenic processes or is a comorbidity parameter associated with severe obesity. Nevertheless, there is no evidence of CCDC80 functioning in liver. Abrogation of $C c d c 80$ in mice has been shown to modulate some circadian clock genes in WAT, skeletal muscle and pancreas at the transcriptomic level, but effects on liver have not been examined (9).

Controversially, despite a higher expression of CCDC80 in VAT of obese patients, no differences were found in serum levels, and circulating CCDC80 was not linked to BMI. Nevertheless, although CCDC80 in human liver is insignificant $(7,8)$, more studies are needed to evaluate the contribution to the serum CCDC80 pool from other tissues, such as skeletal muscle (36) and smooth muscle (37).

\section{CONCLUSION}

Our finding of upregulated CCDC80 expression in the VAT of obese patients indicates that it may be a component of the obesity-altered secretome in this depot. Furthermore, the presence of CCDC80 protein in human serum and its association with systemic metabolic dysfunction suggests that it may be linked to metabolic changes in other organs, since in a cohort with a wide BMI range, increased CCDC80 protein levels were predictive of glucose tolerance derangements. Moreover, both adipose tissue and circulating CCDC80 protein levels were positively associated with inflammatory markers, revealing that CCDC80 is linked to the obesity-associated inflammatory condition. Indeed, in morbid obesity, serum CCDC80 protein levels were associated with inflammation-associated chronic complications, such as FLD and atherosclerosis, suggesting that CCDC80 may be either a pathogenic or comorbidity factor for these diseases.

\section{ACKNOWLEDGMENTS}

This study was supported by the following grants: SAF2012-37480 and SAF2015-65019R from the Spanish Ministerio de Ciencia e Innovación (MCI), FIS-PI14/00228, FIS-P15/01934 and FIS-PI14/00228 from the Fondo de Investigación Sanitaria (FIS), and FLORINASH (VII FP), CIBER de Diabetes y Enfermedades Metabólicas Asociadas (CB07/08/0012) and CIBERobn Fisiopatologia de la Obesidad y Nutrición (CB06/03/010). SF-V acknowledges support from the "Miguel Servet" tenure track program (CPII16/00008) from FIS co-financed by the European Regional Development Fund (ERDF). Sadly, AMG-F passed away before the manuscript was completed.

\section{DISCLOSURE}

The authors declare that they have no competing interests as defined by Molecular Medicine, or other interests that might be perceived to influence the results and discussion reported in this paper.

\section{REFERENCES}

1. Leal VeO, Mafra D. (2013) Adipokines in obesity. Clin Chim Acta. 419:87-94.

2. Gray SL, Vidal-Puig AJ. (2007) Adipose tissue expandability in the maintenance of metabolic homeostasis. Nutr. Rev. 65: S7-12.

3. Rabe K, Lehrke M, Parhofer KG, Broedl UC. (2008) Adipokines and insulin resistance. Mol. Med. 14:741-751.

4. Northcott JM, Yeganeh A, Taylor CG, Zahradka P, Wigle JT. (2012) Adipokines and the cardiovascular system: mechanisms mediating health and disease. Can. J. Physiol. Pharmacol. 90:1029-59.

5. Buechler C, Wanninger J, Neumeier M. (2011) Adiponectin, a key adipokine in obesity related liver diseases. World J. Gastroenterol. 17:2801-11.

6. Aoki K, Sun YJ, Aoki S, Wada K, Wada E. (2002) Cloning, expression, and mapping of a gene that is upregulated in adipose tissue of mice deficient in bombesin receptor subtype-3. Biochem. Biophys. Res. Commun. 290:1282-88.

7. Okada T, et al. (2008) URB is abundantly expressed in adipose tissue and dysregulated in obesity. Biochem. Biophys. Res. Commun. 367: 370-76.
8. Tremblay F, et al. (2009) Bidirectional modulation of adipogenesis by the secreted protein Ccdc80/ DRO1/URB. J. Biol. Chem. 284:8136-47.

9. Tremblay F, et al. (2012) Loss of coiled-coil domain containing 80 negatively modulates glucose homeostasis in diet-induced obese mice. Endocrinology. 153:4290-4303.

10. Liu Y, et al. (2004) URB expression in human bone marrow stromal cells and during mouse development. Biochem. Biophys. Res. Commun. 322:497-507.

11. Recasens M, et al. (2005) An inflammation score is better associated with basal than stimulated surrogate indexes of insulin resistance. J. Clin. Endocrinol. Metab 90:112-16.

12. Moreno-Navarrete JM, et al. (2013) Decreased RB1 mRNA, protein, and activity reflect obesity-induced altered adipogenic capacity in human adipose tissue. Diabetes. 62:1923-31.

13. Serrano M, et al. (2013) Serum lipopolysaccharide-binding protein as a marker of atherosclerosis. Atherosclerosis. 230:223-27.

14. Kleiner DE, et al. (2005) Design and validation of a histological scoring system for nonalcoholic fatty liver disease. Hepatology. 41:1313-21.

15. World Health Organization. (2000) Obesity: Preventing and Managing the Global Epidemic. WHO Technical Report Series 894. 1st ed. Geneva: World Health Organization.

16. Wabitsch M, et al. (2001) Characterization of a human preadipocyte cell strain with high capacity for adipose differentiation. Int. J. Obes. Relat. Metab. Disord. 25:8-15.

17. Chacón M, et al. (2008) Human serum levels of fetal antigen 1 (FA1/Dlk1) increase with obesity, are negatively associated with insulin sensitivity and modulate inflammation in vitro. Int. J. Obes. (Lond). 32:1122-29.

18. Pérez-Pérez R, et al. (2012) Uncovering suitable reference proteins for expression studies in human adipose tissue with relevance to obesity. PLoS One. 7:e30326.

19. Osorio-Conles O, et al. (2011) Plasma PTX3 protein levels inversely correlate with insulin secretion and obesity, whereas visceral adipose tissue PTX3 gene expression is increased in obesity. Am. J. Physiol. Endocrinol. Metab. 301:E1254-61.

20. Taylor PR, et al. (2005) Macrophage receptors and immune recognition. Annu. Rev. Immunol. 23:901-44.

21. Fernández-Real JM, et al. (2011) CD14 modulates inflammation-driven insulin resistance. Diabetes. 60:2179-86.

22. Bommer GT, et al. (2005) DRO1, a gene down-regulated by oncogenes, mediates growth inhibition in colon and pancreatic cancer cells. J. Biol. Chem. 280:7962-75.

23. Matthae S, May S, Hubersberger M, Hauner H, Skurk T. (2013) Protein normalization in different adipocyte models and dependence on cell size. Horm. Metab. Res. 45:572-80.

24. Ibrahim MM. (2010) Subcutaneous and visceral adipose tissue: structural and functional differences. Obes. Rev. 11:11-18. 
25. Insenser M, et al. (2012) A nontargeted proteomic approach to the study of visceral and subcutaneous adipose tissue in human obesity. Mol. Cell. Endocrinol. 363:10-19.

26. Crandall DL, Hausman GJ, Kral JG. (1997) A review of the microcirculation of adipose tissue: anatomic, metabolic, and angiogenic perspectives. Microcirculation. 4:211-32.

27. Catalán V, Gómez-Ambrosi J, Rodríguez A, Frühbeck G. (2012) Role of extracellular matrix remodelling in adipose tissue pathophysiology: relevance in the development of obesity. Histol. Histopathol. 27:1515-28.

28. Lanthier N, Leclercq IA. (2014) Adipose tissues as endocrine target organs. Best Pract. Res. Clin. Gastroenterol. 28:545-58.

29. Grill JI, et al. (2017) Loss of DRO1/CCDC80 results in obesity and promotes adipocyte differentiation. Mol. Cell. Endocrinol. 439:286-96.

30. Bunt JC, Krakoff J, Ortega E, Knowler WC, Bogardus C. (2007) Acute insulin response is an independent predictor of type 2 diabetes mellitus in individuals with both normal fasting and 2-h plasma glucose concentrations. Diabetes Metab. Res. Rev. 23:304-10.

31. Colao A, et al. (2008) Relationships between serum IGF1 levels, blood pressure, and glucose tolerance: an observational, exploratory study in 404 subjects. Eur. J. Endocrinol. 159:389-97.

32. Mócsai A. (2013) Diverse novel functions of neutrophils in immunity, inflammation, and beyond. J. Exp. Med. 210:1283-99.

33. Li Y, Liu L, Wang B, Wang J, Chen D. (2014) Simple steatosis is a more relevant source of serum inflammatory markers than omental adipose tissue. Clin. Res. Hepatol. Gastroenterol. 38:46-54.

34. Targher G, et al. (2008) NASH predicts plasma inflammatory biomarkers independently of visceral fat in men. Obesity (Silver Spring). 16:1394-99.

35. Corrado E, et al. (2010) An update on the role of markers of inflammation in atherosclerosis. J. Atheroscler. Thromb. 17:1-11.

36. Raymond F, et al. (2010) Comparative gene expression profiling between human cultured myotubes and skeletal muscle tissue. BMC Genomics. 11:125.

37. Wang GR, et al. (2013) Steroid-sensitive gene 1 is a novel cyclic GMP-dependent protein kinase I substrate in vascular smooth muscle cells. J. Biol. Chem. 288:24972-83.

Cite this article as: Osorio-Conles Ó, et al. (2017) Adipose tissue and serum CCDC 80 in obesity and its association with related metabolic disease. Mol. Med. 23:225-34. 